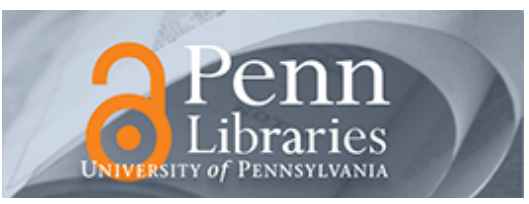

University of Pennsylvania

ScholarlyCommons

June 2003

\title{
Mode Excitation by a Line Source in a Parallel-Plate Waveguide Filled with a Pair of Parallel Double-Negative and Double-Positive Slabs
}

\author{
Andrea Alù \\ University of Pennsylvania, adreaal@seas.upenn.edu \\ Nader Engheta \\ University of Pennsylvania, engheta@seas.upenn.edu
}

Follow this and additional works at: https://repository.upenn.edu/ese_papers

\section{Recommended Citation}

Andrea Alù and Nader Engheta, "Mode Excitation by a Line Source in a Parallel-Plate Waveguide Filled with a Pair of Parallel Double-Negative and Double-Positive Slabs", . June 2003.

Copyright 2003 IEEE. Reprinted from IEEE Antennas and Propagation Society International Symposium, Volume 3, pages 359-362.

Publisher URL: http://ieeexplore.ieee.org/xpl/tocresult.jsp?isNumber=27413\&page=5

This material is posted here with permission of the IEEE. Such permission of the IEEE does not in any way imply IEEE endorsement of any of the University of Pennsylvania's products or services. Internal or personal use of this material is permitted. However, permission to reprint/republish this material for advertising or promotional purposes or for creating new collective works for resale or redistribution must be obtained from the IEEE by writing to pubs-permissions@ieee.org. By choosing to view this document, you agree to all provisions of the copyright laws protecting it.

This paper is posted at ScholarlyCommons. https://repository.upenn.edu/ese_papers/8

For more information, please contact repository@pobox.upenn.edu. 


\title{
Mode Excitation by a Line Source in a Parallel-Plate Waveguide Filled with a Pair of Parallel Double-Negative and Double-Positive Slabs
}

\begin{abstract}
In this paper, we investigate theoretically the excitation of modes by a line source, as well as their modal structure and dispersion, in a parallel-plate waveguide that is filled with a pair of parallel slabs; one being a lossless "double-negative (DNG)" material and the other being a lossless conventional "double-positive (DPS)" medium. Previously, we have shown that such "conjugate" pairing of DNG and DPS materials may lead to reduction of size in one-dimensional cavity resonators and waveguides. The analysis presented here describes the proper modes in such a 1-D parallel plate waveguide, and reveals the possibility of no cut-off thickness for this class of parallel-plate waveguides, which implies that a proper TE or TM mode may always be excited in such a waveguide independent of the overall thickness of the waveguide. The excitation of guided modes by a line source and their power-flow peculiarities are discussed, and the analogy to the concept of "open 1-D cavity" in such a waveguide is mentioned. Some of the interesting features and physical insights regarding the power flow in these guided modes will be presented.

\section{Comments}

Copyright 2003 IEEE. Reprinted from IEEE Antennas and Propagation Society International Symposium, Volume 3, pages 359-362.

Publisher URL: http://ieeexplore.ieee.org/xpl/tocresult.jsp?isNumber=27413\&page=5

This material is posted here with permission of the IEEE. Such permission of the IEEE does not in any way imply IEEE endorsement of any of the University of Pennsylvania's products or services. Internal or personal use of this material is permitted. However, permission to reprint/republish this material for advertising or promotional purposes or for creating new collective works for resale or redistribution must be obtained from the IEEE by writing to pubs-permissions@ieee.org. By choosing to view this document, you agree to all provisions of the copyright laws protecting it.
\end{abstract}




\title{
Mode Excitation by a Line Source in a Parallel-Plate Waveguide Filled with a Pair of Parallel Double- Negative and Double-Positive Slabs
}

\author{
Andrea Alü ${ }^{1,2, *}$ and Nader Engheta ${ }^{1}$ \\ ${ }^{1}$ University of Pennsylvania, Department of Electrical \&Systems Engineering, \\ Philadelphia, Pennsylvania 19104-6390, U.S.A. \\ ${ }^{2}$ Università di Roma Tre, Department of Applied Electronics, \\ via della Vasca Navale, 84 - 00146 Roma, Italy. \\ E-mail: andreaal@ee.upenn.edu, engheta@ee.upenn.edu \\ URL: http://www.ee.upenn.edu/ engheta/ \\ Abstract
}

In this paper, we investigate theoretically the excitation of modes by a line source, as well as their modal structure and dispersion, in a parallel-plate waveguide that is filled with a pair of parallel modal structure and dispersion, in a parallel-plate waveguide that is filled with a pair of paralle
slabs; one being a lossless "double-negative (DNG)" material and the other being a lossless slabs; one being a lossless "double-negative (DNG)" material and the other being a lossless conventional "double-positive (DPS)" medium. Previously, we have shown that such "conjugate" pairing of DNG and DFS materials may lead to reduction of size in one-dimensional cavity resonators and waveguides. The analysis presented here describes the proper modes in such a 1-D parallel plate waveguide, and reveals the possibility of no cut-off thickness for this class of parallelplate waveguides, which implies that a proper TE or TM mode may always be excited in such waveguide independent of the overall thickness of the waveguide. The excitation of guided mode by a line source and their power-flow peculiarities are discussed, and the analogy to the concept of "open 1-D cavity" in such a waveguide is mentioned. Some of the interesting features and physical insights regarding the power flow in these guided modes will be presented.

\section{Introduction}

Research on electromagnetic properties of the "double-negative (DNG)" medium, i.e., the medium in which both permittivity and permeability may possess negative real parts at a certain band of frequency, has become a topic of interests in many research groups around the world. The idea of such a material dates back to Veselago who postulated and studied theoretically the plane wave propagation in such a medium in 1967 [1]. Recently, the research group of Smith, Schultz and Shelby at the University of California San Diego constructed a particulate composite material made of arrays of dipoles and split-ring resonators, and experimentally showed a phenomenon that may be interpreted as an anomalous refraction [2], indicating that such a composite material may be a DNG medium. Since then, a variety of research efforts by many groups has been focused on investigating electromagnetic properties of this class of materials. One such class of problems is the analysis of modes in waveguides and cavities containing DNG media. In an earlier work, Engheta analyzed the electromagnetic fields within a one-dimensional (1-D) parallel-plate cavity resonator in which a pair of lossless DNG and DPS slabs was inserted [3]. There he showed that due to the phase compensation properties of the DNG materials, pairing of DNG and DPS slabs may conceptually provide the possibility of having sub-wavelength thin cavities. Following that work, in [4] he presented a first set of preliminary results and ideas for the guided modes in a parallel-plate waveguide containing these paired slabs. Later in [5], we showed the effects of the anomalous mode coupling between the DNG and DPS open waveguides located parallel to, and in proximity, of each other. Here in the present work, we present our analysis for the detailed modal structures and dispersion of modes excited by a line source in such parallel-plate waveguides. Some other research groups 
have also become interested in certain aspects of waveguides involving DNG media. Among those, one can mention the work reported in [6], [7], and [8]

In the next section, we first present a brief description of modal structures and dispersion characteristics in the parallel-plate DNG-DPS waveguides, and then we discuss mode excitation in such waveguides.

\section{Modal Structure and Dispersion}

The geometry of interest, as shown in Fig. 1, consists of a parallel-plate waveguide with two

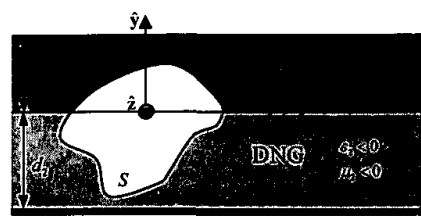

Fig. 1 - Geometry of the problem: a parallel-plate 1-D waveguide filled by a pair of DNG and DPS layers. planes $y=d_{1}$ and $y=-d_{2}$ in the Cartesian coordinate system $(x, y, z)$. Two infinitely extent slabs of lossless materials are inserted in this waveguide. One slab with thickness $d_{1}$ is made of a conventional (DPS) isotropic material with real parameters $\varepsilon_{1}>0$ and $\mu_{1}>0$, while the other slab is assumed to be of a lossless isotropic DNG material with real parameters $\varepsilon_{2}<0$ and $\mu_{2}<0$ at the frequency of interest $\omega$. An infinitely long line source of monochromatic electric current $J(x, y)$ with the cross section $S$ is located inside this waveguide and is parallel with the $z$ axis. A monochromatic $e^{j \omega v}$ excitation is assumed As already noted in [3], this structure supports a resonant mode if

$$
\frac{\tan \left(k_{1} d_{1}\right)}{\tan \left(k_{2} d_{2}\right)}=\frac{k_{1}\left|\mu_{2}\right|}{k_{2}\left|\mu_{1}\right|},
$$

where $k_{i}=\omega \sqrt{\varepsilon_{i} \mu_{i}}$ with $i=1,2$ are the slab wave numbers, real-valued quantities in both DPS and DNG materials. Unlike the conventional cavity resonators, this relation deals with the ratio of tangent functions of the slab thicknesses, and not their sum, which may in principle lead to the possibility of having very thin sub-wavelength resonators, since they are not limited to the standard cut-off thickness $d_{1}+d_{2}>\min \left(\pi / k_{1}, \pi / k_{2}\right)$ that characterizes conventional DPS resonators. When this structure is regarded as a waveguide having a TE guided mode with longitudinal wave number $\beta$, i.e., with $e^{-j \beta_{x}}$ dependence, a corresponding dispersion relation can be found as:

$$
\frac{\tan \left(\sqrt{k_{1}^{2}-\beta^{2}} d_{1}\right)}{\tan \left(\sqrt{k_{2}^{2}-\beta^{2}} d_{2}\right)}=\frac{\left|\mu_{2}\right| \sqrt{k_{1}^{2}-\beta^{2}}}{\left|\mu_{1}\right| \sqrt{k_{2}^{2}-\beta^{2}}} .
$$

which again reveals an unusual feature of this structure, i.e, the fact that the ratio of tangent functions of thicknesses $d_{1}$ and $d_{2}$, not their sum, plays an important role in this modal dispersion. Figure $2 a$ presents the plot of Eq. (2) for a sample waveguide with paired DNG DPS bilayers, showing the minimum total thickness $d_{1}+d_{2}$ in terms of modal longitudinal wave number $\beta$ and the first slab thickness $d_{1}$. For comparison, the plot of the corresponding dispersion relation for a waveguide with a pair of conventional DPS-DPS layers, i.e., $\mu_{1} \sqrt{k_{2}^{2}-\beta^{2}} \tan \left(\sqrt{k_{1}^{2}-\beta^{2}} d_{1}\right)+\mu_{2} \sqrt{k_{1}^{2}-\beta^{2}} \tan \left(\sqrt{k_{2}^{2}-\beta^{2}} d_{2}\right)=0$, is shown in Fig. 2b. The cut at $\beta=0$ in Fig. $2 \mathrm{a}$ indeed satisfies Eq. (1), which is the cavity resonator case. One striking difference between the modal dispersion in the DNG-DPS waveguide (Fig. 2a) and the one for the DPS-DPS waveguide (Fig. 2b) is the fact that when $d_{1}$ approaches zero, the total thickness of the DNG-DPS waveguide required to satisfy Eq. (2) also tends to zero, conceptually implying that there is no cut-off thickness below which no mode can propagate. This is not the 
case for the standard DPS-DPS waveguide, as can been seen from Fig, 2b. What becomes important for such thin DNG-DPS waveguides is the slope of the curve around very small $d_{1}$ and $d_{2}$. In fact, if $d_{1}$ and $d_{2}$ are small enough such that the small-argument approximation can be used for the tangent functions in Eqs. (1) and (2), these equations can be simplified into $d_{1} / d_{2} \cong\left|\mu_{2}\right| /\left|\mu_{1}\right|$, which is a constraint on the ratio between the two slab thicknesses, for given values of material parameters at a fixed frequency. So conceptually, one can have a thin, subwavelength waveguide that can still support a TE (or a TM) propagating mode. As the thicknesses $d_{1}$ and $d_{2}$ become smaller and smaller, while keeping the condition $d_{1} / d_{2} \cong\left|\mu_{2}\right| /\left|\mu_{1}\right|$ satisfied, the value of $\beta$ for the guided mode (which is unique for such thin waveguides) becomes more and more sensitive to slight variations of the structure parameters.
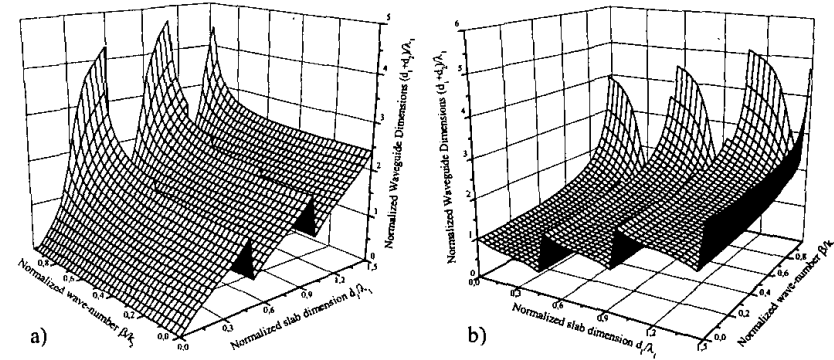

Fig. 2 - Dispersion plots for the waveguide in Fig. 1. Minimum total thickness $\left(d_{1}+d_{2}\right) / \lambda_{1}$ for a waveguide satisfying Eq. (2), in terms of $\beta$ and $d_{1} .\left(\varepsilon_{1}=3, \mu_{1}=3, \varepsilon_{2}=\mp 2, \mu_{2}=\mp 1\right.$, upper signs (DPS-DNG) for Fig. 2a, lower signs (DPS-DPS) for Fig. 2b).

\section{Mode Excitation by a Line Source}

Mode excitation by the line source $J(x, y)$ in this DNG-DPS parallel-plate waveguide can be treated by exploiting the mode orthogonality. A $z$-oriented line source excites TE modes with the following expressions for the transverse electric field:

$$
\boldsymbol{E}_{n}=E_{0 n} \boldsymbol{e}_{n}=E_{0 n} \hat{z} \begin{cases}e^{-j \beta_{n} x} \sin \left(k_{t+12} d_{2}\right) \sin \left(k_{t n 1}\left(d_{1}-y\right)\right) & 0 \leq y<d_{1} \\ e^{-j \beta_{n} x} \sin \left(k_{t+1} d_{1}\right) \sin \left(k_{t n 2}\left(y+d_{2}\right)\right) & -d_{2}<y<0\end{cases}
$$

where $\beta_{n}$ 's satisfy Eq. (2) and $k_{r i i}=\sqrt{k_{i}^{2}-\beta_{n}^{2}}$ with $i=1,2$. The expressions for the transverse and longitudinal components of the magnetic field $H_{n}\left(\equiv E_{o n} h_{n}\right)$ can be straightforwardly obtained using the Maxwell equations. It can be shown that these guided modes in the DNGDPS waveguide are orthogonal, and therefore distinct modes with differing $\beta$ 's carry power without any power exchange. At a fixed frequency and for a given set of material parameters $\varepsilon_{1}, \mu_{1}, \varepsilon_{2}$, and $\mu_{2}$ and slab thicknesses $d_{1}$ and $d_{2}$, equation (2) has a finite number (say $M$ ) of real solutions for $\beta$, which correspond to the propagating modes in the structure. At a sufficiently large distance from the source region, evanescent modes can be neglected and the real power flow is associated only with the $M / 2$ guided propagating modes with real $\beta_{n}$ s, carrying power in the $\hat{x}$ direction and the other $M / 2$ guided modes with real $-\beta_{n}$ s, carrying power in the $-\hat{\boldsymbol{x}}$ direction. It is worth noting that each guided mode has a real component of the Poynting vector along the $\mathrm{x}$ axis that is parallel with $\beta$ in the DPS slab and antiparallel with $\beta$ in the DNG slab. This is similar in the case of Zenneck wave propagating at the 
interface between a DNG half space and a DPS half space discussed in [9]. Therefore, one needs to consider a "net" power flow, that is the algebraic sum, or equivalently the difference between the magnitude, of the power fluxes over the cross sections of DPS and of DNG slabs. Such a net power for each guided mode is expressed by

$$
\iint_{\text {guide enos section }} \frac{1}{2} \operatorname{Re}\left[\boldsymbol{E}_{n} \times \boldsymbol{H}_{n}^{*}\right] \cdot \hat{x} d y=\frac{\left|E_{0 n}\right|^{2} \beta_{n}}{4 \omega}\left(\frac{d_{1}-\frac{\sin \left(2 k_{t n 1} d_{1}\right)}{2 k_{t n 1}}}{\mu_{1} \sin ^{-2}\left(k_{t n 2} d_{2}\right)}+\frac{d_{2}-\frac{\sin \left(2 k_{t n 2} d_{2}\right)}{2 k_{t n 2}}}{\mu_{2} \sin ^{-2}\left(k_{t n 1} d_{t}\right)}\right)
$$

The sign of this net power flow may be opposite to the sign of $\beta_{n}$ (depending on the sign of the expression in the parentheses in the right hand side), implying a backward guided mode with phase and group velocities being antiparallel. Using the Lorentz reciprocity theorem, we can find the magnitude $E_{o n}$ for the $n^{\text {th }}$ mode as follows

$$
E_{a n}= \pm\left(\int_{\text {Cross section of Source }} J(x, y) \cdot \boldsymbol{e}_{n}^{*} e^{j \beta_{n} x} d x d y\right) /\left(2 \int_{\text {guide cross section }} \operatorname{Re}\left[\boldsymbol{e}_{n} \times h_{n}^{*}\right] \cdot \hat{x} d y\right)
$$

where the choice in the sign depends on whether the guided mode is a forward or backward wave. An analogous concept for guided waves with backward power flow was also studied previously in circular waveguides loaded inhomogeneously with dielectric rods or ferrites (e.g., [10]). One of the differences between the current DPS-DNG parallel-plate waveguides and those circular waveguides with backward-wave structures is the complete separation between the two opposite power fluxes here, one being in the DPS layer and the other in the DNG layer, which potentially presents some interesting possibilities for microwave applications. Finally, i should be noted that as a particular case it may be possible to have the net power flow for a guided mode zero in Eq. (4), even though $\beta \neq 0$. In such a case, the power flux in the DPS layer is equal in magnitude and opposite in direction to the power flux in the DNG layer, implying that the waveguide is effectively acting as an "open cavity". One such scenario may happen when the DPS and DNG layers are so-called "perfectly matched", i.e., $\varepsilon_{1}=-\varepsilon_{2}$, $\mu_{1}=-\mu_{2}, d_{1}=d_{2}$. Aside from this special case, very thin waveguides capable of supporting a propagating mode do carry nonzero net-power, thus potentially allowing miniaturization of guided wave components.

\section{References}

[1] V. G. Vesclago, "The electrodynamics of substances with simultancously negative values of $\varepsilon$ and $\mu$, Soviet Physics Uspekhi, vol. 10, no. 4, pp. 509-514, 1968, [Usp. Fiz. Nauk, vol. 92, pp. 517-526, 1967].

[2] R. A. Shelby, D. R. Smith, S. Schultz, "Experimental verification of a negative index of refraction," Sclence, vol. 292, no. 5514, pp. 77-79, April 6, 2001

[3] N. Engheta, "An idea for thin subwavelength cavity resonators using metamatcrials with negative permittivity and permcability, "IEEE Antennas and Wireless Propagation Letters, Vol. 1, No. 1, pp. 10-13, 2002.

[4] N. Engheta, "Guided Waves in Paired Dielectric-Metamaterial with Negative Permittivity and Perncability Layers" presented at the USNC-URSI National Radio Science Meeting, in Boulder, Colorado, Jan. 9-12, 2002. Page 66. with Negative Permittivity and Permeability," presented at the IEEE-Nano'2002 conference, Washington DC, August with Negative Permittivity

26-28, 2002, pp. 233-234. [6] A. Topa, "Contradircenional intcrat August 17-24, 2002, paper number 1878 in Union of $R$ Re

August 17-24, 2002, paper number 1878 in the CD digest.
[7] I. S. Nefedov and S. A. Trectyakov, "Theoretical Study of Wavcguiding Structures Containing BackwardWave Materials, "in the XXVII General Assembly of International Union of Radio Science (URSI GA'02) in Maastricht, The Netherlands, August 17-24, 2002, paper number 1074 in the $\mathrm{CD}$ digest.

[8] C. Caloz, C.-C. Chang, and T. Itoh, "Full-wave verification of the fundamental propertics of left-handed materials in waveguide configurations," Journal of Applied Physics, Vol. 90, No. II, pp. 5483-5486, December 2001. [9] A. Alù, N. Engheta, "Radiation from a traveling-wave current shect at the interface between a conventional matcrial and a metamaterial with negative permittivity and permeability," Microwave and Optical Technology Letters, Vol. 35, No. 6, Deccmber 20, 2002, pp. 460-463. [10] P. J. B. Clarticoats, "Circular-waveguide backward-wave structures," Proc. IEE, vol. 110, no. 2, Fcb. 1963, 